\title{
Morita equivalence for positive Hochschild homology and dihedral homology
}

\author{
Marco Farinati ${ }^{1}$ - Andrea Solotar ${ }^{* 1}$
}

\begin{abstract}
In this paper we prove the invariance of positive Hochschild homology and dihedral homology with respect to hermitian Morita equivalence between involutive algebras. We also define the notion of hermitian $k$-congruence and prove some results on Morita invariance of $\mathrm{HH}_{*}^{+}$and $H D_{*}$ in this context.
\end{abstract}

\section{Introduction}

Let us consider two rings $A$ and $B$. They are Morita equivalent if and only if their module categories are equivalent. The Morita invariance of $H H_{*}$ was proved by Dennis-Igusa [2].

If $A$ and $B$ are equipped with involutions $\omega_{A}$ and $\omega_{B}$, we may consider their positive Hochschild homology $\mathrm{HH}_{*}^{+}$and their dihedral homology $H D_{*}$ as well as the notion of hermitian Morita equivalence.

One of the main purposes of this paper is to show that $\mathrm{HH}_{*}^{+}$and $H \mathrm{D}_{*}$ are invariant under hermitian Morita equivalence, which is proved in Theorem 2.7 .

If $A$ and $B$ are Morita equivalent and $A$ is involutive, it is always possible to define an involution on $B$ such that the equivalence is an hermitian one. Also, it may happen that $H_{*}^{+}(A) \neq H H_{*}^{+}(B)$ even when they are Morita equivalent in the usual sense, so, for involutive algebras, $H H_{*}^{+}$and $H D_{*}$ are finer invariants than $H H_{*}$ and $H C_{*}$.

\footnotetext{
${ }^{*}$ Research member of CONICET (Argentina)

${ }^{1}$ Dto. de Matemática Facultad de Cs. Exactas y Naturales. Universidad de Buenos Aires. Ciudad Universitaria Pab I. 1428 - Buenos Aires - Argentina e-mail: asolotar@mate.dm.uba.armfarinat@mate.dm.uba.ar
} 
In the second part of this paper, we consider the following fact: for a given $A$, the functor $H_{*}(A,-)$ (resp. $H_{*}^{+}(A,-)$ ) is applied at the category of $A$-bimodules (resp. compatible $A$-bimodules). The proof of Morita invariance of Hochschild homology makes explicit use of the isomorphisms given by the Morita equivalence. So we study what happens under a weaker condition: the equivalence of the bimodule categories.

We use, after Schack [8], the notion of $k$-congruence, and we define the notion of hermitian $k$-congruence. We show, in Theorem 3.6, that $H H_{*}$ and $H_{*}^{+}$are also invariant under $k$-congruences (resp. hermitian $k$-congruences). Finally, we show that if $A$ is an involutive $B$ - Azumaya algebra, where $\mathrm{B}$ is a commutative involutive $k$-algebra, then $H H_{*}^{+}(A / k)=$ $H H_{*}^{+}(B / k)$.

We want to thank M. Vigué and the referee for helpful comments. 


\section{Hermitian Morita invariance}

We first recall some definitions and fix notation. Let $k$ be an arbitrary commutative ring with $1, A$ and $B$ two unital $k$-algebras. We will denote with ${ }_{A} \bmod$ the category of left $A$-modules, $\bmod _{A}$ the category of right $A$ modules, ${ }_{A} \bmod _{B}$ the category of bimodules (with left action of $A$ and a right action of $B$ ) and ${ }_{A}$ bimod the category of $A$-bimodules.

Definition 2.1 $A$ and $B$ are Morita equivalent if and only if there exist two bimodules ${ }_{A} P_{B}$ and ${ }_{B} Q_{A}$ such that $P \otimes_{B} Q \cong A$ and $Q \otimes_{A} P \cong B$ as bimodules, with the usual bimodule structure of $A$ and $B$.

This definition is equivalent to the following facts:

- The categories ${ }_{A} \bmod$ and ${ }_{B} \bmod$ are equivalent.

- The categories $\bmod _{A}$ and $\bmod _{B}$ are equivalent.

The equivalences are given by:

$$
\begin{aligned}
& Q \otimes_{A}-::_{A} \bmod \rightarrow_{B} \bmod \\
& -\otimes_{A} P: \bmod _{A} \rightarrow \bmod _{B}
\end{aligned}
$$

As a consequence, ${ }_{A}$ bimod and ${ }_{B}$ bimod are equivalent, by the functor:

$$
P \otimes_{B}-\otimes_{B} Q:_{B} \text { bimod } \rightarrow_{A} \text { bimod }
$$

Remark(on Def. 2.1): If ${ }_{A} \bmod$ and ${ }_{B} \bmod$ are equivalent by a functor $F$, then take $Q=F(A)$ and $P=F^{-1}(B)$.

It is well known that if $A$ and $B$ are Morita equivalent, then $H_{*}(A, M) \cong$ $H_{*}\left(B, Q \otimes_{A} M \otimes_{B} P\right)$ and $H C_{*}(A) \cong H C_{*}(B)$ (idem for cohomology). Suppose now that $A$ and $B$ are equipped with involutions of algebras $\omega_{A}$ and $\omega_{B}$, and let $M$ be an $A$-bimodule which has an involution $\omega_{M}$ compatible with the involution of $A$, namely, $\omega_{M}: M \rightarrow M$ is an additive map such that $\omega_{M}^{2}=i d_{M}$ and $\omega_{M}\left(a m a^{\prime}\right)=\omega_{A}\left(a^{\prime}\right) \omega_{M}(m) \omega_{A}(a)$, for $m \in M, a, a^{\prime} \in A$.

Example: $M=A \otimes A^{o p}$ is a compatible $A$-bimodule, where $\omega_{M}: A \otimes A^{o p} \rightarrow A \otimes A^{o p}$ is defined by $\omega_{M}\left(a \otimes a^{\prime}\right)=\omega_{A}\left(a^{\prime}\right) \otimes \omega_{A}(a)$.

The $A$-bimodule $M$ is equivalent to a left $A^{e}=A \otimes_{k} A^{o p}$-module, via $\left(a \otimes a^{\prime}\right) m=a m a^{\prime}$. The category of compatible $A$-bimodules is actually 
a category of modules over a certain ring $R$ defined as follows: $R$ is the twisted group algebra $R=\mathbb{Z} / 2 \mathbb{Z} \rtimes A^{e}$, where the generator $\sigma$ of $\mathbb{Z} / 2 \mathbb{Z}$ acts by $\sigma(a \otimes b)=\omega_{A} b \otimes \omega_{A} a$. Precisely, $R=k[\mathbb{Z} / 2 \mathbb{Z}] \otimes A^{e}=A^{e} \oplus A^{e} \sigma$ equipped with the following multiplication: $\left(e_{1}+f_{1} \sigma\right)\left(e_{2}+f_{2} \sigma\right)=\left(e_{1} e_{2}+f_{1} \sigma\left(f_{2}\right)\right)+$ $\left(e_{1} f_{2}+f_{1} \sigma\left(e_{2}\right)\right) \sigma$ The fact that $R_{R}$ mod $=(($ compatible $A$-bimodules $))$ follows from the observation that $R$ is the endomorphism ring of the projective generator $A^{e} \oplus A^{e}$ with involution $(e, f) \rightarrow(\sigma(f), \sigma(e))$. Explicitly, any compatible $A$-bimodule $M$ is an $R$-module by $(e+f \sigma) m=e m+f \omega_{M}(m)$.

At this step there are at least three questions to answer:

- Is $H_{*}^{+}(A, M)$ isomorphic to $H_{*}^{+}\left(B, Q \otimes_{A} M \otimes_{A} P\right)$ ?

- Is $H D_{*}(A)$ isomorphic to $H D_{*}(B)$ ?

- If $M$ is a compatible $A$-bimodule, can we define an involution on $Q \otimes_{A}$ $M \otimes_{A} P$ so that we obtain a compatible $B$-bimodule?

Remark: It is necessary to talk about bimodules because if not, the compatibility condition with the involution of the ring makes no sense.

An hermitian Morita theory for algebras with involution was introduced in [3] and generalized by Hahn in [5].

According to the definitions of Hahn, and fixing notation, we have:

Definition 2.2 $A$ and $B$ are hermitian Morita equivalent, if they are Morita equivalent (in the usual sense) and (with the notations of definition 2.1):

1. $P$ and $Q$ are $k$-symmetric (i.e. $\lambda p=p \lambda \forall \lambda \in k, p \in P$, idem for $Q$ )

2. There exist $\mu: P \otimes_{B} Q \rightarrow A$ and $\tau: Q \otimes_{A} P \rightarrow B$ isomorphisms of $A$-bimodules and $B$-bimodules respectively such that:

(a) $\mu(p \otimes q) p^{\prime}=p \tau\left(q \otimes p^{\prime}\right)$ and

(b) $\tau(q \otimes p) q^{\prime}=q \mu(p \otimes q) \forall p, p^{\prime} \in P, q, q^{\prime} \in Q$

3. There exists an additive bijection $\Theta: P \rightarrow Q$ such that

(a) $\mu\left(p \otimes \Theta\left(p^{\prime}\right)\right)=\omega_{A}\left(\mu\left(p^{\prime} \otimes \Theta(p)\right)\right)$

(b) $\tau\left(\Theta(p) \otimes p^{\prime}\right)=\omega_{B}\left(\tau\left(\Theta\left(p^{\prime}\right) \otimes p\right)\right)$ and

(c) $\Theta(a p b)=\omega_{B}(b) \Theta(p) \omega_{A}(a) \forall a \in A, b \in B, p, p^{\prime} \in P, q, q^{\prime} \in Q$ 
A pair of bimodules ${ }_{A} P_{B}$ and ${ }_{B} Q_{A}$ satisfying these properties will be called invertible bimodules.

Hahn shows that under these hypothesis the categories ((hermitian $\left.{ }_{A} m o d\right)$ ) and $\left(\left(\right.\right.$ hermitian $\left.\left.{ }_{B} \bmod \right)\right)$ are equivalent, where ((hermitian $\left.\left.{ }_{A} m o d\right)\right)$ is the category whose objects are $A$-modules equipped with a $\omega_{A}$-sesquilinear form.

Another interpretation of this definition will be given by proposition 2.3. We will now fix some notation.

Let

$$
\begin{aligned}
& F A:_{A} \bmod \rightarrow A^{o p} \bmod =\bmod _{A} \\
& F B:_{B} \bmod \rightarrow_{B^{o p}} \bmod =\bmod _{B}
\end{aligned}
$$

be the functors induced by the involutions $\omega_{A}$ and $\omega_{B}$. Namely, if $M$ is a left $A$-module then $F A(M)=M$ as abelian group, and the right $A$-action is given by:

$$
m . a=\omega_{A}(a) . m \forall m \in M, a \in A
$$

If $f:{ }_{A} M \rightarrow_{A} N$ then $F A(f)=f$.

Now, if $A$ and $B$ are Morita equivalent we then have two possible functors from ${ }_{A}$ mod to ${ }_{B}$ mod. One is the usual $Q \otimes_{A}-$, and the other one is the composition:

$$
{ }_{A} \bmod \stackrel{F A}{\longrightarrow} \bmod _{A} \stackrel{-\otimes_{A} P}{\longrightarrow} \bmod _{B} \stackrel{F B}{\longrightarrow}{ }_{B} \bmod
$$

Proposition 2.3 The following facts are equivalent:

1. $\exists \Theta: P \rightarrow Q \mathbb{Z}$-isomorphism such that

$$
\Theta(a . p . b)=\omega_{B}(b) \Theta(p) \omega_{A}(a) \forall a \in A, p \in P, b \in B
$$

2. The functors $Q \otimes_{A}-$ and $F B\left(-\otimes_{A} P\right) F A$ are naturally equivalent.

\section{Proof:}

1. $\Rightarrow 2$.

We observe that $\Theta(a p b)=\omega_{B}(b) \Theta(p) \omega_{A}(a) \forall a \in A, b \in B$ and $p \in P$ if and only if $\Theta^{-1}(b q a)=\omega_{A}(a) \Theta^{-1}(q) \omega_{B}(b) \forall a \in A, b \in B$ and $q \in Q$.

Let $\eta_{M}: Q \otimes_{A} M \rightarrow F B\left(F A(M) \otimes_{A} P\right)$ defined by

$$
\eta_{M}\left(q \otimes_{A} m\right)=m \otimes_{A} \Theta^{-1}(q)
$$


$\eta_{M}$ is a well-defined $B$-module morphism because of the properties of $\Theta$ with respect to scalars.

$\eta_{M}$ is an isomorphism with inverse $m \otimes_{A} p \mapsto \Theta(p) \otimes_{A} m$.

The naturality of $\eta_{M}$ is easily checked.

2. $\Rightarrow 1$.

We remark that $\omega_{A}: F A(A) \rightarrow A$ is an isomorphism of $A$-bimodules:

$$
\omega_{A}\left(a^{\prime} \cdot a \cdot a^{\prime \prime}\right)=\omega_{A}\left(\omega_{A}\left(a^{\prime \prime}\right) \cdot a \cdot \omega_{A}\left(a^{\prime}\right)\right)=a^{\prime} \cdot \omega_{A}(a) \cdot a^{\prime \prime}
$$

where the action on the first term is the action on $F A(A)$, and the usual one on the others.

When $M=A$, the natural transformation gives us a morphism:

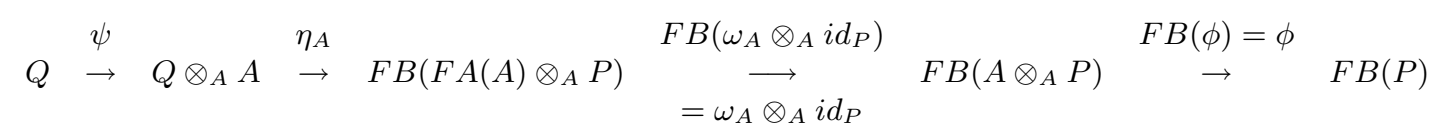

We define $\Theta^{-1}$ as the composition:

$$
\Theta^{-1}=\phi\left(\omega_{A} \otimes_{A} i d_{P}\right) \eta_{A} \psi: Q \rightarrow F B(P)
$$

$\Theta^{-1}$ is composition of isomorphisms of $B$-modules, then a $\mathbb{Z}$-isomorphism with the property $\Theta^{-1}(b q)=b \Theta^{-1}(q)=\Theta^{-1}(q) \omega_{B}(b)$ (the action is on $F B(P))$.

If $a \in A$ we have the following:

$$
\begin{gathered}
\Theta^{-1}(q a)=\phi\left(\omega_{A} \otimes_{A} i d_{P}\right) \eta_{A} \psi(q a)=\phi\left(\omega_{A} \otimes_{A} i d_{P}\right) \eta_{A}\left(q \otimes_{A} a\right)= \\
=\phi\left(\omega_{A} \otimes_{A} i d_{P}\right) \eta_{A}\left(i d_{Q} \otimes_{A} \times a\right)\left(q \otimes_{A} 1\right)
\end{gathered}
$$

where $\times a$ is the right multiplication by $a$. By the naturality of $\eta_{A}$, the following diagram is commutative:

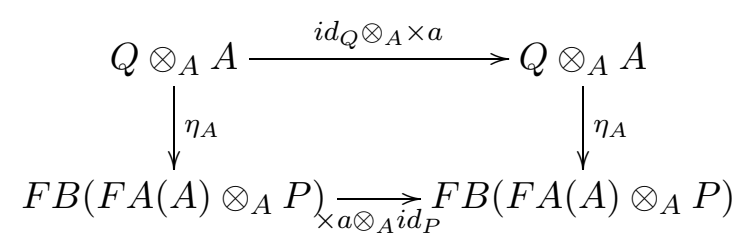

Then:

$\phi\left(\omega_{A} \otimes_{A} i d_{P}\right) \eta_{A}\left(i d_{Q} \otimes_{A} \times a\right)\left(q \otimes_{A} 1\right)=\phi\left(\omega_{A} \otimes_{A} i d_{P}\right)\left(\times a \otimes_{A} i d_{P}\right) \eta_{A}\left(q \otimes_{A} 1\right)=$ 


$$
\begin{gathered}
=\phi\left(\omega_{A} \otimes_{A} i d_{P}\right)\left(\times a \otimes_{A} i d_{P}\right)\left(\omega_{A} \otimes_{A} i d_{P}\right)^{-1}\left(\omega_{A} \otimes_{A} i d_{P}\right) \eta_{A}\left(q \otimes_{A} 1\right)= \\
=\phi\left(\omega_{A} \times a \omega_{A}^{-1} \otimes_{A} i d_{P}\right)\left(\omega_{A} \otimes_{A} i d_{P}\right) \eta_{A}\left(q \otimes_{A} 1\right)
\end{gathered}
$$

but $\omega_{A}(\times a) \omega_{A}^{-1}\left(a^{\prime}\right)=\omega_{A}\left(\omega_{A}\left(a^{\prime}\right) . a\right)=\omega_{A}(a) \cdot a^{\prime}$ then $\omega_{A}(\times a) \omega_{A}^{-1}=$ $\omega_{A}(a) \times$ where $\omega_{A}(a) \times: A \rightarrow A,\left(a^{\prime} \mapsto \omega_{A}(a) \cdot a^{\prime}\right)$.

So:

$$
\begin{gathered}
\Theta^{-1}(q a)=\phi\left(\omega_{A}(a) \times \otimes_{A} i d_{P}\right)\left(\omega_{A} \otimes_{A} i d_{P}\right) \eta_{A}\left(q \otimes_{A} 1\right)= \\
=\phi\left(\omega_{A}(a) \times \otimes_{A} i d_{P}\right) \phi^{-1} \phi\left(\omega_{A} \otimes_{A} i d_{P}\right) \eta_{A}\left(q \otimes_{A} 1\right)=\phi\left(\omega_{A}(a) \times \otimes_{A} i d_{P}\right) \phi^{-1} \Theta^{-1}(q)= \\
=\phi\left(\omega_{A}(a) \times \otimes_{A} i d_{P}\right)\left(1 \otimes_{A} \Theta^{-1}(q)\right)=\phi\left(\omega_{A}(a) \otimes_{A} \Theta^{-1}(q)\right)=\omega_{A}(a) \Theta^{-1}(q)
\end{gathered}
$$

We obtain $\Theta(a p b)=\omega_{B}(b) \Theta(p) \omega_{A}(a) \forall a \in A, b \in B$ and $p \in P$.

Lemma 2.4 If $M$ is an A-bimodule which has an involution $\omega_{M}$ compatible with the involution $\omega_{A}$ of $A$, then $Q \otimes_{A} M \otimes_{A} P$ has, in a natural way, an involution compatible with the involution $\omega_{B}$ of $B$.

\section{Proof:}

Let $\omega: Q \otimes_{A} M \otimes_{A} P \rightarrow Q \otimes_{A} M \otimes_{A} P$ be defined by:

$$
\omega(q \otimes m \otimes p)=\Theta(p) \otimes \omega_{M}(m) \otimes \Theta^{-1}(q)
$$

It is easy to verify that $\omega$ satisfies the required properties.

Definition 2.5 In the conditions of definition 2.2, we shall say that

$$
\left(A, \Theta::_{A} P_{B} \rightarrow_{B} Q_{A}, B\right) \text { is an hermitian set of equivalence data }
$$


Lemma 2.6 If $\Theta, \mu, \tau$ satisfy conditions 1. 2. and 3. of definition 2.2, then $\mu$ and $\tau$ are compatible with the involutions,

i.e.: $\mu(\omega(p \otimes q))=\omega_{A}(\mu(p \otimes q))$ and $\tau\left(\omega^{\prime}(q \otimes p)\right)=\omega_{B}(\tau(q \otimes p))$.

where:

$\omega: P \otimes_{A} Q \rightarrow P \otimes_{A} Q \omega(p \otimes q)=\Theta^{-1}(q) \otimes \Theta(p)$

$\omega^{\prime}: Q \otimes_{B} P \rightarrow Q \otimes_{A} P \omega^{\prime}(q \otimes p)=\Theta(p) \otimes \Theta^{-1}(q)$

Proof: Immediate.

Examples:

1. Let $B=M_{n}(A)$ with $\left(\omega_{B}(m)\right)_{i j}=\omega_{A}\left(m_{j i}\right)$ if $m \in M_{n}(A), P=A^{1 \times n}$ (row vectors), $Q=A^{n \times 1}$ (column vectors) and $\Theta: P \rightarrow Q$ given by $\Theta\left(a_{1}, \ldots, a_{n}\right)=\left(\omega_{A}\left(a_{1}\right), \ldots, \omega_{A}\left(a_{n}\right)\right)^{t}$.

The conditions of definition 2.2 are easily verified.

2. Let $A$ be a $k$-algebra with involution, $B=A, \omega_{B}(a)=\gamma \omega_{A}(a) \gamma$ where $\gamma \in A$ is such that $\gamma^{2}=1, \Theta: A \rightarrow A, \Theta(x)=\gamma \omega_{A}(x)$.

3. Let $\left(A, \omega_{A}\right)$ be an involutive $k$-algebra, $P$ a finitely generated projective $A$-module, $B=\operatorname{End}_{A}(P)$. Then $P \in_{A} \bmod _{B}$ and if $B$ is equipped with the involution induced by the involution of $M_{n}(A)$ we have an hermitian set of equivalence data.

4. Let $M$ be a $C^{\infty}$ locally compact manifold, and $G$ a discrete group, acting on $M$ without fixed points.

Let $A=C^{\infty}(M / G)$ be the algebra of complex-valued $C^{\infty}$-functions that vanish at the infinity. $B=C^{\infty}(M) \rtimes G$.

We consider the involution $\omega_{A}$ in $A$ given by complex conjugation, and the involution $\omega_{B}$ in $B$ given by:

$$
\omega_{B}(\phi, g)=\left(g^{-1} \phi^{*}, g^{-1}\right)
$$

Where $\phi \in C^{\infty}(M), g \in G$, and if $m \in M: \phi^{*}(m)=\phi(m)^{*}$ (the complex conjugation). The action of $G$ in $C^{\infty}(M)$ is given by $(h \phi)(m)=\phi\left(h^{-1} m\right)$ $\forall m \in M, h \in G$.

We take $P=Q=C^{\infty}(M)$, equipped with the following structure:

1. Left $A$-module by: $(f \phi)(m)=f([m]) \phi(m)$

2. Right $B$-module by: $\left(\phi\left(\phi_{1}, g\right)\right)(m)=\phi(g m) \phi_{1}(g m)$ 
3. Right $A$-module as in 1 .

4. Left $B$-module by: $\left.\left(\left(\phi_{1}, g\right) \phi\right)(m)=\phi_{1}(m) \phi_{(} g^{-1} m\right)$

where $f \in C^{\infty}(M / G) ; \phi_{1}, \phi \in C^{\infty}(M) ; m \in M, g \in G$ and $[m]$ denote the class of $m$ in $M / G$.

We consider the additive morphism $\Theta: P \rightarrow Q=P$ given by complex conjugation. We check that $\Theta(a p b)=\omega_{B}(b) \Theta(p) \omega_{A}(a) \forall a \in A, b \in B$, $p \in P$.

Finally we define the bimodule isomorphisms $\mu: P \otimes_{B} Q \rightarrow A$ and $\tau: Q \otimes_{A} P \rightarrow B$ by:

$$
\tau(\phi, \psi)=\sum_{x \in G}\left(F_{x}, x\right) \quad \text { where } F_{x} \in C^{\infty}(M), F_{x}(m)=\phi(m) \psi\left(x^{-1} m\right)
$$

and

$$
\mu(\phi, \psi)([m])=\sum_{x \in G} \phi\left(x^{-1} m\right) \psi\left(x^{-1} m\right) \quad\left(\phi, \psi \in C^{\infty}(M)\right)
$$

1. $\tau\left(\Theta(p) \otimes p^{\prime}\right)=\omega_{B}\left(\tau\left(\Theta\left(p^{\prime}\right) \otimes p\right)\right)$

2. $\mu(p \otimes q) p^{\prime}=p \tau\left(q \otimes p^{\prime}\right)$ and

3. $\tau(q \otimes p) q^{\prime}=q \mu\left(p \otimes q^{\prime}\right) \forall p, p^{\prime} \in P, q, q^{\prime} \in Q$

Then it will follow from next Theorem and 2.8 that $H H_{*}^{+}(A) \cong H H_{*}^{+}(B)$ and $H D_{*}(A) \cong H D_{*}(B)$.

\section{Remarks:}

1. These last equalities show the usefulness of computations of $\mathrm{HH}_{*}^{+}$and $H D_{*}$ of crossed products.

2. The last example is a particular case of the notion of "Strong Morita equivalence" for $C^{*}$-algebras defined by Rieffel [7].

Theorem 2.7 In the conditions of Def.2.2 and Lemma 2.6, if $1 / 2 \in k$ :

$$
H_{*}^{+}(A, M) \cong H_{*}^{+}\left(B, Q \otimes_{A} M \otimes_{A} P\right)
$$

Proof: During this proof, we will write $\otimes$ instead of $\otimes_{A}$ or $\otimes_{B}$ when the meaning of $\otimes$ is clear by the context.

At the beginning, the proof is similar to the proof of Morita invariance of Hochschild homology. Namely, we know that there exist 
$\left\{p_{1}, \ldots p_{s}\right\} \subset P,\left\{p_{1}^{\prime}, \ldots, p_{m}^{\prime}\right\} \subset P,\left\{q_{1}, \ldots, q_{s}\right\} \subset Q,\left\{q_{1}^{\prime}, \ldots, q_{m}^{\prime}\right\} \subset Q$ such that:

$$
\mu\left(\sum_{i=1}^{s} p_{i} \otimes q_{i}\right)=1_{A} \quad \tau\left(\sum_{j=1}^{m} q_{j}^{\prime} \otimes p_{j}^{\prime}\right)=1_{B}
$$

Then we define a morphism of complexes:

$$
\begin{aligned}
& \psi: M \otimes A^{\otimes *} \rightarrow\left(Q \otimes_{A} M \otimes_{A} P\right) \otimes B^{\otimes *} \\
& \phi:\left(Q \otimes_{A} M \otimes_{A} P\right) \otimes B^{\otimes *} \rightarrow M \otimes A^{\otimes *}
\end{aligned}
$$

as in $([6]$, p.19).

For $\psi$ (the other is similar), one has:

$$
\begin{aligned}
& \psi_{n}\left(m \otimes a_{1} \otimes \ldots \otimes a_{n}\right)=\sum_{1 \leq j_{i} \leq s}\left(q_{j_{0}} \otimes m \otimes p_{j_{1}} \tau\left(q_{j_{1}} \otimes a_{1} \otimes p_{j_{2}}\right) \otimes \ldots \otimes \tau\left(q_{j_{n}} \otimes a_{n} \otimes p_{j_{0}}\right)\right) \\
& \omega\left(\psi_{n}\left(m \otimes a_{1} \otimes \ldots \otimes a_{n}\right)\right)= \\
&=(-1)^{n(n+1) / 2} \sum_{1 \leq j_{i} \leq s} \omega\left(q_{j_{0}} \otimes m \otimes p_{j_{1}}\right) \otimes \omega_{B}\left(\tau\left(q_{j_{n}} \otimes a_{n} \otimes p_{j_{0}}\right)\right) \otimes \ldots \otimes \omega_{B}\left(\tau\left(q_{j_{1}} \otimes a_{1} \otimes p_{j_{2}}\right)\right) \\
&=\left.(-1)^{n(n+1) / 2} \sum_{1 \leq j_{i} \leq s} \Theta\left(p_{j_{1}}\right) \otimes \omega_{M}(m) \otimes \Theta^{-1}\left(q_{j_{0}}\right) \otimes \tau \omega\left(q_{j_{n}} \otimes a_{n} \otimes p_{j_{0}}\right)\right) \otimes \ldots \otimes \tau \omega\left(q_{j_{1}} \otimes a_{1} \otimes p_{j_{2}}\right) \\
&=(-1)^{n(n+1) / 2} \sum_{1 \leq j_{i} \leq s} \Theta\left(p_{j_{1}}\right) \otimes \omega_{M}(m) \otimes \Theta^{-1}\left(q_{j_{0}}\right) \otimes \tau\left(\Theta\left(a_{n} p_{j_{0}}\right) \otimes \Theta^{-1}\left(q_{j_{n}}\right)\right) \otimes \ldots \otimes \tau\left(\Theta\left(a_{1} p_{j_{2}}\right) \otimes \Theta^{-1}\left(q_{j_{1}}\right)\right) \\
&=(-1)^{n(n+1) / 2} \sum_{1 \leq j_{i} \leq s} \Theta\left(p_{j_{1}}\right) \otimes \omega_{M}(m) \otimes \Theta^{-1}\left(q_{j_{0}}\right) \otimes \tau\left(\Theta\left(p_{j_{0}}\right) \omega_{A}\left(a_{n}\right) \otimes \Theta^{-1}\left(q_{j_{n}}\right)\right) \otimes \ldots \otimes \tau\left(\Theta\left(p_{j_{2}}\right) \omega_{A}\left(a_{1}\right) \otimes \Theta^{-1}\left(q_{j_{1}}\right)\right)
\end{aligned}
$$

If $\left(m \otimes a_{1} \otimes \ldots \otimes a_{n}\right) \in\left(M \otimes A^{\otimes *}\right)^{+}$, then

$$
\left(m \otimes a_{1} \otimes \ldots \otimes a_{n}\right)=(-1)^{n(n+1) / 2} \omega_{M}(m) \otimes \omega_{A}\left(a_{n}\right) \otimes \ldots \otimes \omega_{A}\left(a_{1}\right)
$$

and then:

$\psi_{n}\left(\omega\left(m \otimes a_{1} \otimes \ldots \otimes a_{n}\right)\right)=\psi_{n}\left((-1)^{n(n+1) / 2} \omega_{M}(m) \otimes \omega_{A}\left(a_{n}\right) \otimes \ldots \otimes \omega_{A}\left(a_{1}\right)\right)$

So, $\sum_{1 \leq j_{i} \leq s}\left(q_{j_{0}} \otimes m \otimes p_{j_{1}} \otimes \tau\left(q_{j_{1}} \otimes a_{1} p_{j_{2}}\right) \otimes \ldots \otimes \tau\left(q_{j_{n}} \otimes a_{n} p_{j_{0}}\right)\right)=$

$\left.=(-1)^{n(n+1) / 2} \sum_{1 \leq j_{i} \leq s}\left(q_{j_{0}} \otimes \omega_{M}(m) \otimes p_{j_{1}}\right) \otimes \tau\left(q_{j_{n}} \otimes \omega_{A}\left(a_{n}\right) p_{j_{0}}\right)\right) \otimes \ldots \otimes \tau\left(q_{j_{1}} \otimes \omega_{A}\left(a_{1}\right) p_{j_{2}}\right)$ 
As $\tau\left(\sum_{i=1}^{s} q_{j_{i}} \otimes p_{j_{i}}\right)=1_{B}$, then $1_{B}=\tau\left(\sum_{i=1}^{s} \Theta\left(p_{j_{i}}\right) \otimes \Theta^{-1}\left(q_{j_{i}}\right)\right)$

And as $\tau$ is an isomorphism, $\sum_{i=1}^{s} q_{j_{i}} \otimes p_{j_{i}}=\sum_{i=1}^{s} \Theta\left(p_{j_{i}}\right) \otimes \Theta^{-1}\left(q_{j_{i}}\right)$.

If we define $\psi_{n}^{\Theta}$ by the same formula but replacing the sets $\left\{p_{1}, \ldots, p_{s}\right\}$ and $\left\{q_{1}, \ldots, q_{s}\right\}$ by $\left\{\Theta^{-1}\left(q_{1}\right), \ldots, \Theta^{-1}\left(q_{s}\right)\right\}$ and $\left\{\Theta\left(p_{1}\right), \ldots, \Theta\left(p_{s}\right)\right\}$ respectively, we have that:

$$
\omega \psi_{n}=\psi_{n}^{\Theta} \omega
$$

As a consequence we have the following commutative diagrams:

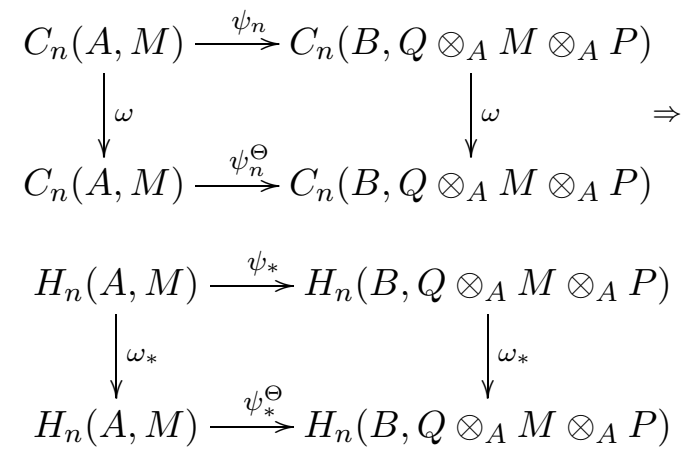

Loday shows that $\psi \phi$ is homotopic to the identity by defining an explicit homotopy $h_{i}$ (and the same for $\phi \psi$ ), then $\psi_{*}=\phi_{*}^{-1}$.

If one considers $h_{i}^{\Theta}$ defined by the same formula as $h_{i}$ but replacing $p_{i}^{\prime}$ by $\Theta^{-1}\left(q_{i}\right)$ and $q_{i}^{\prime}$ by $\Theta^{-1}\left(p_{i}\right)$, then $\psi^{\Theta} \phi$ is homotopic to the identity, so $\psi_{*}^{\Theta}=\phi_{*}^{-1}$. As a consequence $\psi_{*}^{\Theta}=\psi_{*}$.

This proves that

$$
H_{n}^{+}(A, M) \cong \psi_{*} H_{n}^{+}\left(B, Q \otimes_{A} M \otimes_{A} P\right)
$$

Remark: Sometimes we may have $\Theta\left(q_{i}\right)=p_{i}$, for all $i$ (for example in the case $B=M_{n}(A)$, , see [6]). If this is the case, the hypothesis that $1 / 2 \in k$ is not necessary, but the adequate version of positive Hochschild homology in this case, denoted $H^{i n v}$ is the homology of the double complex-called homotopy colimit- having all columns equal to the Hochschild complex with 2-periodic row boundary map $1 \pm \omega$. As now the chain map giving Morita invariance commutes with the operator $\omega$, it follows that Morita invariance also holds for this homology.

Proposition 2.8 Under the same hypothesis of Theorem 2.7, $H D_{*}(A) \cong$ $H D_{*}(B)$ 
Proof: We have explicit maps $H D_{*}(A) \rightarrow H D_{*}(B), H H_{*}^{+}(A) \rightarrow H H_{*}^{-}(B)$ and $H H_{*}^{-}(A) \rightarrow H H_{*}^{+}(B)$ that induce commutative diagrams between the Connes's long exact sequence in dihedral homology for $A$ and $B$. From Theorem 2.7 and a similar version for $H H_{*}^{-}$, we deduce that $H D_{*}(A) \rightarrow H D_{*}(B)$ is an isomorphism, using a five-Lemma argument.

\section{Equivalences between bimodule categories}

The proof of Morita invariance of $H H_{*}$ and $H C_{*}$ makes use of the existence of an equivalence between the categories of $A$-bimodules and $B$-bimodules, and the existence of invertible bimodules ${ }_{B} P_{A}$ and ${ }_{A} Q_{B}$ allows us to build explicitly the bimodule equivalence.

The equivalence between the categories ${ }_{A}$ bimod and ${ }_{B}$ bimod is in general weaker than the equivalence between ${ }_{A} \bmod$ and ${ }_{B} \bmod$. In fact, ${ }_{A} \bmod$ and ${ }_{B} \bmod$ are equivalent if and only if $\bmod _{A}$ and $\bmod _{B}$ are equivalent. Both imply that ${ }_{A}$ bimod is equivalent to ${ }_{B}$ bimod, but if for example $A$ is a (non commutative) ring such that $\otimes_{A}$ is not symmetric, then the converse does not hold.

Example: Let $\mathbb{H}$ denote the quaternionic numbers, i.e. $\mathbb{H}$ is the real algebra generated by $i, j, k$ with relations $i^{2}=j^{2}=k^{2}=-1$ and $i j=k=$ $-j i ; j k=i=-k j ; k i=j=-i k$. Since the set of real numbers is a field, every ring Morita equivalent to it is a matrix ring with real coefficients. $\mathbb{H}$ has dimension 4 (as a real vector space), if it is Morita equivalent to the real numbers then it must be isomorphic to $M_{2}(\mathbb{R})$, but that is not possible because $\mathbb{H}$ is a division ring. Nevertheless $\mathbb{H} \cong \mathbb{H}^{o p}$ and $\mathbb{H} \otimes_{\mathbb{R}} \mathbb{H} \cong M_{4}(\mathbb{R})$ (isomorphism of real algebras) and $M_{4}(\mathbb{R})$ is Morita equivalent to the real

numbers. That shows that the category of $\mathbb{H}$-bimodules $=\mathbb{H} \otimes \mathbb{R} \mathbb{H}^{o p}$-modules is equivalent to the category of real vector spaces, but the category of $\mathbb{H}$ modules is not.

This is a a particular example of the following situation: Consider an algebra $A$ over a commutative ring $R$. $A$ is called an Azumaya algebra over $R$ if the category of $A$-bimodules is Morita equivalent to the category of $R$ modules. Examples of Azumaya algebras are Clifford algebras of even ranked quadratic modules, in particular the quaternion algebra $\mathbb{H}$. A result due to Schack [8] and also proved in [1] states that if $R$ is a commutative algebra over a field $k$ and $A$ is an Azumaya algebra over $R$, then $H H_{*}(A / k)=$ 
$H H_{*}(R / k)$. We shall study this situation for involutive $k$-algebras at the end of this Section.

In [8] Schack defines the notion of $k$-congruence (weak and strong) between two unital $k$-algebras $A$ and $B$ as follows:

Definition 3.1 An equivalence of categories $T:_{A}$ bimod $\rightarrow_{B}$ bimod is called a $k$-congruence if and only if:

1. (weak) $T(A) \cong \gamma$ (where $\gamma$ denotes the isomorphism)

2. (strong) $T\left(M \otimes_{A} N\right) \cong T(M) \otimes_{B} T(N)$

Remark: Strong $k$-congruence implies weak $k$-congruence.

Schack shows that every Morita equivalence induces a strong

$k$-congruence, and claims that if $A$ and $B$ are such that there exists a weak $k$-congruence between them, then $H^{*}(A, M) \cong H^{*}(B, T(M))$ (where $M$ is any $A$-bimodule).

Let now $\left(A, \omega_{A}\right)$ and $\left(B, \omega_{B}\right)$ be two unital involutive $k$-algebras. We are interested in the bimodule equivalences $T$ which preserve involutions.

We know that every equivalence between ${ }_{A} \operatorname{bimod}={ }_{A^{e}} \bmod$ and $B_{B^{e}} \bmod$ is obtained by tensoring with a module $R \in{ }_{B} \bmod _{A^{e}}$, where $R=T\left(A^{e}\right)$. Let's suppose that $R$ is equipped with an involution $\omega_{R}$ compatible with $A$ and $B$ (i.e. $\omega_{R}\left(a r a^{\prime}\right)=\omega_{A}\left(a^{\prime}\right) \omega_{R}(r) \omega_{A}(a)$ and $\omega_{R}\left(b r b^{\prime}\right)=\omega_{B}\left(b^{\prime}\right) \omega_{R}(r) \omega_{A}(b)$ $\forall a, a^{\prime} \in A, b, b^{\prime} \in B$ and $\left.r \in R\right)$; then if $M$ is a compatible $A^{e}$-module, $T(M)=R \otimes_{A^{e}} M$ has a well-defined involution $\omega_{R \otimes_{A^{e}} M}=\omega_{R} \otimes_{A^{e}} \omega_{M}$ that makes $T(M)$ into a compatible $B^{e}$-module.

In this context we make the following:

Definition 3.2 $T$ is an involutive $k$-congruence if and only if it is a (weak) $k$-congruence and $\omega_{B}(b)=\gamma\left(\omega_{A}(a) \otimes_{A^{e}} \omega_{R}(r)\right) \forall b \in B$, where $\gamma\left(a \otimes_{A^{e}} r\right)=b$. We write then $T\left(\left(A, \omega_{A}\right)\right)=\left(B, \omega_{B}\right)$, $(\gamma$ is the isomorphism of the definition 3.1).

Proposition 3.3 Every hermitian equivalence $\bar{T}:_{A} \bmod \rightarrow_{B} \bmod$ induces an involutive $k$-congruence

$T:\left(\left(\right.\right.$ compatible ${ }_{A}$ bimod $\left.)\right) \rightarrow\left(\left(\right.\right.$ compatible ${ }_{B}$ bimod $\left.)\right)$. i.e. a $k$-congruence $T:{ }_{Z / 2 Z} \rtimes_{A^{e}}-\bmod \rightarrow_{Z / 2 Z} \rtimes_{B^{e}}-\bmod$

Proof: An hermitian Morita equivalence is given by a pair of invertible bimodules ${ }_{A} P_{B}$ and ${ }_{B} Q_{A}$ and an additive map $\Theta: P \rightarrow Q$ satisfying the 
conditions of 2.2. Then we can take $R=Q \otimes_{k} P$ with the involution $\omega_{R}: Q \otimes_{k} P \rightarrow Q \otimes_{k} P, \omega_{R}(q \otimes p)=\Theta(p) \otimes \Theta^{-1}(q)$. The induced equivalence is then $T(M)=R \otimes_{A^{e}} M=\left(Q \otimes_{k} P\right) \otimes_{A^{e}} M \cong Q \otimes_{A} M \otimes_{A} P$.

$T(A) \cong Q \otimes_{A} A \otimes_{A} P \cong Q \otimes_{A} P \cong B$, and we can easily check that $T\left(A, \omega_{A}\right)=\left(B, \omega_{B}\right)$.

As Schack has already remarked, every $k$-linear functor $T:{ }_{A}$ bimod $\rightarrow_{B}$ bimod defines a morphism between the Yoneda extensions $\operatorname{Ext}_{A^{e}}^{*}(-,-)$ and $\operatorname{Ext}_{B^{e}}^{*}(T(-), T(-))$, applying $T$ to every bimodule in each class of extension, which is clearly an isomorphism when $T$ is an equivalence. In particular, when $A$ and $B$ are $k$-projective and $T(A) \cong B$ we have $H^{*}(A, M) \cong H^{*}(B, T(M))$ for every $A$-bimodule $M$. In the case of involutive $k$-algebras we want to show that $H^{*+}(A, M) \cong H^{*+}(B, T(M))$ for every compatible $A$-bimodule $M$, and analogously for homology.

We will suppose, until the end of this section, that $A$ and $B$ are both $k$-projective, and if $T$ is an equivalence between the categories of $A^{e} \bmod$ and $B^{e} m o d$, let $B_{B^{e}} R_{A^{e}}$ and ${ }_{A^{e}} S_{B^{e}}$ be bimodules such that $R \otimes_{A^{e}} S \cong B^{e}$, $S \otimes_{B^{e}} R \cong A^{e}$ (bimodule isomorphisms) and $T=R \otimes_{A^{e}}(-)$. The condition that $T$ is a $k$-congruence implies that $R \otimes_{A^{e}} A \cong B$ as $B^{e}$-module. Now we require also that $A \otimes_{A^{e}} S \cong B$.

The condition of projectivity of $A$ and $B$ over $k$ allows us to compute $H_{*}(A, M)$ as $\operatorname{Tor}_{*}^{A^{e}}(A, M)$, using $A^{e}$-projective resolutions of $A$.

If $\left(P_{n}, d_{n}\right)_{n \geq 0}$ is an $A^{e}$-projective resolution of $A$, the fact that $-\otimes_{A^{e}} S$ is also an equivalence implies that $\left(P_{n} \otimes_{A^{e}} S, d_{n} \otimes i d_{S}\right)_{n \geq 0}$ is a $B^{e}$-projective resolution of $A \otimes_{A^{e}} S \cong B$. Then $H_{*}(B, T(M))=\operatorname{Tor}_{*}^{B^{e}}\left(B, R \otimes_{A^{e}} M\right)$ is the homology of the complex

$\left(P_{n} \otimes_{A^{e}} S \otimes_{B^{e}} R \otimes_{A^{e}} M, d_{n} \otimes i d_{S} \otimes i d_{R} \otimes i d_{M}\right)_{n \geq 0} \cong$ $\cong\left(P_{n} \otimes_{A^{e}} A^{e} \otimes_{A^{e}} M, d_{n} \otimes i d_{A^{e}} \otimes i d_{M}\right)_{n \geq 0} \cong\left(P_{n} \otimes_{A^{e}} M, d_{n} \otimes i d_{M}\right)_{n \geq 0}$. The homology of this last complex computes $\operatorname{Tor}_{*}^{A^{e}}(A, M)$. So we have proved the following:

Proposition 3.4 With the notations as above, if $A$ and $B$ are $k$-projective and $A \otimes_{A^{e}} S \cong B$ as a B-bimodule, then $H_{*}(A, M) \cong H_{*}(B, T(M))$

Let us now return to the case where $A$ and $B$ are involutive algebras and $T$ is an involutive $k$-congruence.

Suppose we have an $A^{e}$-projective resolution of $A\left(P_{n}, d_{n}\right)_{n \geq 0}$ such that every $P_{n}$ is a compatible $A^{e}$-module, equipped with an involution such that $\omega_{P} . d=d . \omega_{P}$. In this situation we will say that we have an involutive 
projective resolution, which is the same thing that a projective resolution in the category of $\mathbb{Z} / 2 \mathbb{Z} \rtimes A^{e}$-modules.

Lemma 3.5 If $M$ is a compatible A-bimodule, then:

$$
H_{*}^{i n v}(A, M)=\operatorname{Tor}_{*}^{Z / 2 Z \rtimes A^{e}}(A, M)
$$

Proof: Let $C^{\prime}(A)$ be the Hochschild resolution of $A$ as an $A^{e}$-module. Define $y^{\prime}: C_{n}^{\prime}(A) \rightarrow C_{n}^{\prime}(A)$ as $y^{\prime}\left(a \otimes a_{1} \otimes \ldots \otimes a_{n} \otimes a^{\prime}\right)=(-1)^{n(n+1) / 2}\left(\omega_{A} a^{\prime} \otimes\right.$ $\left.\omega_{A} a_{n} \otimes \ldots \otimes \omega_{A} a_{1} \otimes \omega_{A} a\right)$. Let $\mathbb{Z} / 2 \mathbb{Z}$ act on $C_{*}^{\prime}(A) \oplus C_{*}^{\prime}(A)$ by $\left(x_{1}, x_{2}\right) \rightarrow$ $\left(y^{\prime}\left(x_{2}\right), y^{\prime}\left(x_{1}\right)\right)$, and form the corresponding homotopy colimit as in Section 2. The result is a double complex whose total complex is a projective $\mathbb{Z} / 2 \mathbb{Z} \rtimes A^{e}$-module in each degree. Moreover, tensoring the latter complex over $\mathbb{Z} / 2 \mathbb{Z} \rtimes A^{e}$ with $M$ is exactly the complex that computes $H_{*}^{i n v}(A, M)$ as defined before. This concludes the proof.

Theorem 3.6 Under the same notations and hypothesis as in 3.5:

$$
H_{*}^{i n v}(A, M) \cong H_{*}^{i n v}(B, T(M))
$$

Proof: It is enough to remark the following facts:

- The standard Hochschild resolution of $A$, equipped with the involution $y^{\prime}$ defined in the previous lemma is a $\mathbb{Z} / 2 \mathbb{Z} \rtimes A^{e}$ - projective resolution.

- $H_{*}^{i n v}(A, M)=\operatorname{Tor}_{*}^{Z / 2 Z \rtimes A^{e}}(A, M)$.

- With the same argument as in proposition 3.4, one obtains an involutive $B^{e}$-projective resolution of $B$.

So, the theorem is a corollary of the above discussion.

\section{Remarks}

1. An analogous proof shows that the same holds for cohomology.

2. If $A$ is a commutative $k$-projective algebra, we can define an explicit morphism $f_{*}: H H_{*}(A) \rightarrow H H_{*}(B)$ such that $f_{0}$ is an isomorphism and $f_{*}$ makes the long exact sequences commutative as follows. 
Let $r_{0} \in R$ be such that $\gamma\left(r_{0} \otimes_{A^{e}} 1_{A}\right)=1_{B}$, and let $f_{n}: H H_{n}(A) \rightarrow H H_{n}(B)$ be defined by $f_{n}\left(\left[a_{0} \otimes \ldots \otimes a_{n}\right]\right)=\left[\gamma\left(r_{0} \otimes a_{0}\right) \otimes \ldots \otimes \gamma\left(r_{0} \otimes a_{n}\right)\right]$ $f_{n}$ is well-defined because $\gamma\left(r_{0} \otimes a\right) \gamma\left(r_{0} \otimes a^{\prime}\right)-\gamma\left(r_{0} \otimes a a^{\prime}\right)$ is in general a linear combination of terms of the type $\gamma\left(r_{i} \otimes a_{i}^{\prime \prime}\right)$ with $a_{i}^{\prime \prime} \in[A, A]$, but $A$ is commutative.

Then, since in the above situation $H H_{*}$ is an universal functor, we know that $H H_{*}(A) \cong H H_{*}(B)$, and the commutativity of $f_{*}$ with the involutions shows that $H H_{*}^{+}(A) \cong f_{*} H H_{*}^{+}(B)$.

Finally, looking at the case of involutive Azumaya algebras, we remark that, as a corollary of 3.6 and Schack's Theorem 3 [8], we have:

Proposition 3.7 Let $B$ be an involutive and commutative $k$-algebra, and let $A$ be an Azumaya B-algebra Then any hermitian $B$-congruence $T:_{B}$ bimod $\rightarrow A$ bimod induces an isomorphism

$$
H_{*}^{i n v}(B,-) \rightarrow H_{*}^{i n v}(A, T(-))
$$

\section{References}

[1] G.Cortiñas - Ch. Weibel: Homology of Azumaya algebras. Proc. AMS, 121(1) (1994), p.53-55.

[2] K. Dennis - K. Igusa: Hochschild homology and the second obstruction for pseudo-isotopy. Lect. Notes Math. 966 (1982), p. 7-58.

[3] A. Fröhlich - Mc. Evett: Forms over rings with involution, J. of Alg.12 (1969), p. 79-104.

[4] M. Gerstenhaber: On the deformations of rings and algebras. Ann. of Math. 79 (1964), p. 59-103.

[5] A. Hahn: An hermitian Morita theorem for algebras with antistructure. J.of Alg. 93 (1985), p. 215-235.

[6] J. L. Loday: Cyclic homology. Der Grund. Math. Wiss. 301 Springer Verlag 1992.

[7] M. Rieffel: Morita equivalence for operator algebras. Proc. of Symposia in Pure Math. Vol 38 (1982) Part I, p. 285-298. 
[8] S. D. Schack: Bimodules, the Brauer group, Morita invariance, and cohomology. J. of P. and A. Alg. 80 (1992), p. 315-325. 